\title{
Magnetic field dependence of the proximity induced triplet superconductivity at ferromagnet/superconductor interfaces
}

\author{
Yoav Kalcheim, Israel Felner and Oded Millo \\ Racah Institute of Physics and the Hebrew University Center for Nanoscience and \\ Nanotechnology, The Hebrew University of Jerusalem, \\ Jerusalem 91904, Israel \\ Tal Kirzhner and Gad Koren \\ Department of Physics, Technion - Israel Institute of Technology, Haifa 32000, Israel \\ Angelo di Bernardo, Mehmet Egilmez, Mark G. Blamire, and Jason W. A. Robinson \\ Department of Material Science and Metallurgy, University of Cambridge, 27 \\ Charles Babbage Road, Cambridge, CB3 OFS, United Kingdom
}

\begin{abstract}
Long-ranged superconductor proximity effects recently found in superconductorferromagnetic (S-F) systems are generally attributed to the formation of triplet-pairing correlations due to various forms of magnetic inhomogeneities at the $\mathrm{S}-\mathrm{F}$ interface. In order to investigate this conjecture within a single $\mathrm{F}$ layer coupled to a superconductor, we performed scanning tunneling spectroscopy on bilayers of $\mathrm{La}_{2 / 3} \mathrm{Ca}_{1 / 3} \mathrm{MnO}_{3}$ (LCMO) ferromagnetic thin-films grown on high temperature superconducting films of $\mathrm{YBa}_{2} \mathrm{Cu}_{3} \mathrm{O}_{7}$ $\delta(\mathrm{YBCO})$ or $\mathrm{Pr}_{1.85} \mathrm{Ca}_{0.15} \mathrm{CuO}_{4}(\mathrm{PCCO})$ under various magnetic fields. We find a strong correlation between the magnitude of superconductor-related spectral features measured on the LCMO layer and the degree of magnetic inhomogeneity controlled by the external magnetic field. This corroborates theoretical predictions regarding the role played by magnetic inhomogeneities in inducing triplet-pairing at S-F interfaces.
\end{abstract}


Proximity effects in superconductor-ferromagnet (S-F) hybrids became a subject of intensive research in recent years, partly due to observations of long-range spin-polarized supercurrents in S-F-S Josephson junctions, signifying the appearance of a proximityinduced spin-triplet pairing order in F. It is well known that ferromagnetism and spinsinglet superconductivity are two inimical orders, as one is associated with electrons of parallel spin alignment and the other with Cooper pairs formed from electrons with antiparallel aligned spins. Consequently, the proximity effect (PE) in S-F junctions is expected to be short ranged due to the exchange field $\left(E_{e x}\right)$ in $\mathrm{F}$ that acts to dephase the two electrons of opposite sign of spin. ${ }^{1,2}$ This leads to a very short penetration depth of superconducating order into $\mathrm{F}$, on a length scale of $\xi_{F}=\sqrt{\hbar D / 2 E_{e x}}$, where $D$ is the diffusivity in F. For strong ferromagnets, $\xi_{F} \sim 1 \mathrm{~nm}$, much shorter than the typical penetration depth of S order into a normal metal, $\xi_{N}=\sqrt{\hbar D / k_{B} T}$, that can be as large as $100 \mathrm{~nm}$ at low temperatures.

Despite the expected short coherence length of Cooper pairs in F materials, over the past decade experiments on S-F-S Josephson junctions with the half metallic ferromagnet ( $\mathrm{HMF})\left(\mathrm{CrO}_{2}\right),{ }^{3-5}$ intermetallic $\left(\mathrm{Cu}_{2} \mathrm{MnAl}\right),{ }^{6}$ and metallic $(\mathrm{Co})^{7,8}$ barriers have revealed evidence of supercurrents in $\mathrm{F}$ barriers much thicker than $\xi_{F}$. Equal-spin Andreev reflections and long range coherent transport were found also in $\mathrm{YBa}_{2} \mathrm{Cu}_{3} \mathrm{O}_{7-\delta}$ $/ \mathrm{La}_{2 / 3} \mathrm{Ca}_{1 / 3} \mathrm{MnO}_{3}$ (YBCO/LCMO) multilayers through the observation of McMillanRowell resonances, ${ }^{9}$ and signatures of a triplet in $\mathrm{La}_{0.7} \mathrm{Sr}_{0.3} \mathrm{MnO}_{3} / \mathrm{YBCO} / \mathrm{La} 0.7 \mathrm{Sr}_{0.3} \mathrm{MnO}_{3}$ trilayers appeared in the magnetic field evolution of the critical temperature and conductance spectra. ${ }^{10}$ In addition, scanning tunneling microscopy and spectroscopy (STM and STS) experiments ${ }^{11,12}$ provided evidence of long ranged PE in half-metallic F (HMF) LCMO films, for which $\xi_{F}$ is estimated to be smaller than $1 \mathrm{~nm}\left(E_{e x} \sim 3 \mathrm{eV}\right.$ and the Fermi velocity is rather small $\left.7 \times 10^{7} \mathrm{~cm} / \mathrm{s}\right) .{ }^{13}$ Superconducting-related features were observed in the tunneling spectra measured on LCMO layers as thick as $\sim 30 \mathrm{~nm}$, much larger than $\xi_{F}$, in bilayers of $\mathrm{LCMO} /(100) \mathrm{YBCO}$ and $\mathrm{LCMO} / \mathrm{Pr}_{1.85} \mathrm{Ca}_{0.15} \mathrm{CuO}_{4}$ (PCCO). ${ }^{11,12}$ However, such effects were not found for $\mathrm{LCMO} /(001) \mathrm{YBCO}$ bilayers, ${ }^{14}$ as discussed in the Supplemental Material. ${ }^{15}$ 
The mechanisms proposed for the long ranged PE involve the conversion of singlet state Cooper pairs into an equal-spin triplet state in $\mathrm{F}$, which is insensitive to the ferromagnetic exchange field. It has been suggested that such a conversion process can occur due to some form of magnetic inhomogeneity such as a domain wall ${ }^{16}$ or a spinactive F-S interface. ${ }^{17,18}$ Bergeret, Volkov and Efetov ${ }^{19}$ showed theoretically that rotation of the magnetization inside a domain wall, for example, can promote the formation of an equal-spin triplet state. Similarly, in a mechanism proposed by Eschrig et al. ${ }^{17}$ an F-S interface can give rise to a triplet state with zero projection in spin space on the magnetization axis of the interface. If the magnetizations at the F-S interface and inside the F layer are non-collinear, the triplet state at the interface has a non-zero spin-projection on the F-layer interior magnetization, and the corresponding equal-spin triplet component decays in $\mathrm{F}$ on a length scale of $\xi_{N}$. For an excellent review on this issue, see Ref. ${ }^{20}$. Furthermore, these mechanisms predict that the induced superconductor order-parameter (OP) can have an orbital symmetry which is even ( $s$ - or $d$-wave) or odd ( $p$ - or $f$-wave), maintaining Fermionic anti-symmetry by a suitable odd or even dependence of the Matsubara frequency. Because anisotropic OPs (e.g., $d$-wave or $p$-wave) are sensitive even to non-magnetic disorder it is expected that the superconducting correlations should predominately be carried into the $\mathrm{F}$ by an odd frequency $s$-wave component while lower symmetry OPs should appear less abundantly. Our previous STM measurements on $\mathrm{LCMO} / \mathrm{YBCO}^{11}$ and $\mathrm{LCMO} / \mathrm{PCCO}^{12}$ bilayers are consistent with this prediction; the tunneling spectra revealed mainly proximity gaps over large regions on the LCMO surface, and to a much lesser extent zero-bias conductance peaks (ZBCPs), which are known to be associated with anisotropic sign-changing OPs such as $p$ - and $d$-wave.

In spite of ample evidence for PE-induced triplet-pairing at S-F interfaces and some recent experiments which clarify the role of magnetic inhomogeneity in engineered magnetic multilayers, ${ }^{7,8,21}$ it is still not well established experimentally whether the intrinsic magnetic inhomogeneity within a single ferromagnetic material proximity coupled to a superconductor plays an important role in generating equal-spin triplet components, as suggested by theory. ${ }^{17,18}$ Here we report STM and STS measurements in magnetic field showing that the degree of magnetic homogeneity within a single $\mathrm{F}$ layer can greatly affect the magnitude of the superconducting-related spectral features in LCMO 
proximity coupled to YBCO or PCCO. Our findings show that magnetization misalignment between different regions around S-F interfaces is key to generating long-range PE.

STM and STS measurements were performed at $4.2 \mathrm{~K}$ on two series of epitaxial bilayers grown by pulsed laser deposition, consisting of LCMO films with thicknesses

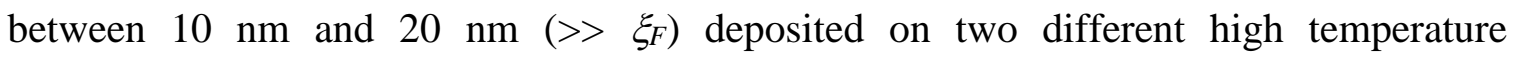
superconductor films. The first are optimally (hole) doped $a$-axis (100)YBCO films, 135 $\mathrm{nm}$ thick, grown on (100) $\mathrm{SrTiO}_{3}$ (STO) substrates, and the second are $300 \mathrm{~nm}$ thick (electron-doped) PCCO films, deposited on (001) $\mathrm{NdGaO}_{3}$ substrates. In both cases, the $\mathrm{S}$ and LCMO films were grown consecutively without breaking vacuum, showing a magnetic transition at $\sim 250 \mathrm{~K}$ and superconducting transitions at $88 \mathrm{~K}$ (YBCO) and $17 \mathrm{~K}$ (PCCO). For further details see Ref. ${ }^{15}$. We also acquired tunneling spectra on control samples of bare 15-nm-thick and 20-nm-thick LCMO grown directly on substrates of (100) $\mathrm{LaAlO}_{3}$ (LAO). The corresponding results are described in Ref. ${ }^{15}$.

The spatial distribution of the superconducting-related spectral features found in our previous studies $^{11,12}$ in zero magnetic field was reproduced in the samples studied here. Namely, superconducting related spectral features (mainly gaps) were observed over large regions, hundreds of nanometers in size (much larger than the width of domain walls in LCMO, $\sim 20 \mathrm{~nm})^{22}$. Figure 1 presents tunneling $d I / d V$ versus $V$ spectra featuring gaps in the quasi-particle density of states (DoS) on a 17-nm-LCMO/YBCO bilayer as a function of applied magnetic field. The spectra were acquired without changing the STM (current and voltage) settings except for the magnetic field, which was applied perpendicular to the S-F interface. Before applying a magnetic field the spectrum is gapped with normalized zero bias conductance $(\mathrm{ZBC})$ of 0.73 . When applying a magnetic field of $75 \mathrm{mT}$ the gap becomes more pronounced with the normalized $\mathrm{ZBC}$ decreasing to 0.64 , indicating a reduction in the quasi-particle DoS at the Fermi energy. It is important to note that when applying a magnetic field perpendicular to the easy axis, which lies in-plane in our sample, the saturation field of the LCMO films is found to be $\sim 300 \mathrm{mT} .{ }^{23}$ By further increasing the magnetic field the $\mathrm{ZBC}$ rises until it reaches 0.85 at $360 \mathrm{mT}$. The inset of Fig. 1 shows the normalized ZBC taken from each curve as function of magnetic field. 


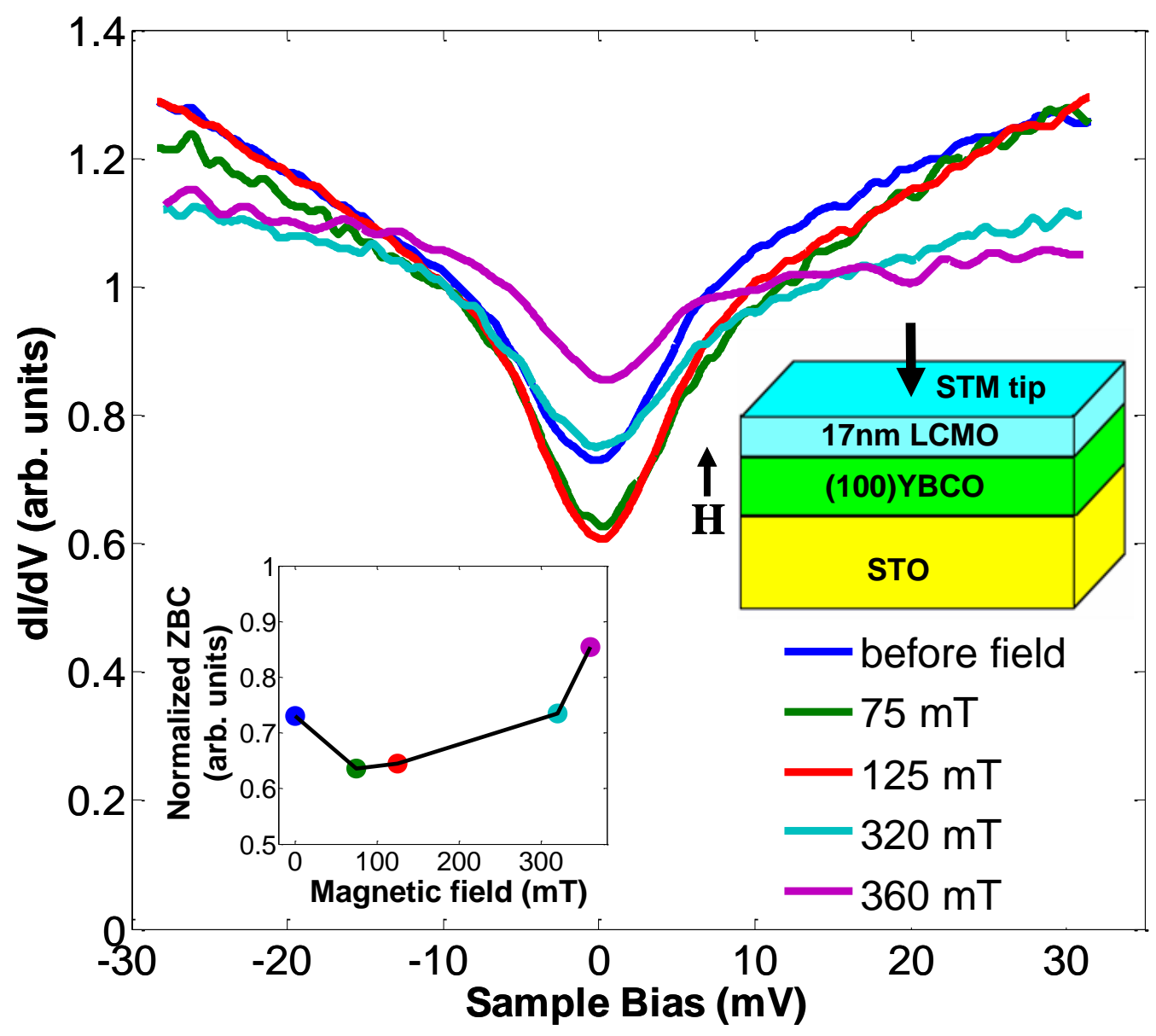

Figure 1: Normalized (to the gap edge) tunneling $d I / d V$ versus $V$ spectra at $4.2 \mathrm{~K}$ acquired on a 17-nm-LCMO/(100)YBCO sample in different magnetic fields applied perpendicular to the interface. Left inset: The normalized zero-bias conductance of the superconductor proximity gap as a function of magnetic field. Note the non-monotonic dependence of the ZBC on magnetic field. Right inset: Scheme of the bilayer film and measurement configuration. The error bars are smaller than the dot size.

A similar non-monotonic dependence of the proximity induced gap on magnetic field was found for 14-nm-LCMO/YBCO bilayer, as shown in Fig. 2. The inset of Fig. 2 shows that the magnitude of the gap hardly changes when the applied field is reduced from 180 $\mathrm{mT}$ to zero and then increased to $150 \mathrm{mT}$. This behavior implies that the magnetization distribution remained intact (at least in the measurement region) during the field recycling 
process, which took place over a time scale of $\sim 1 \mathrm{~min}$. The effect of such magnetic viscosity is discussed in the Supplemental Material. ${ }^{15}$

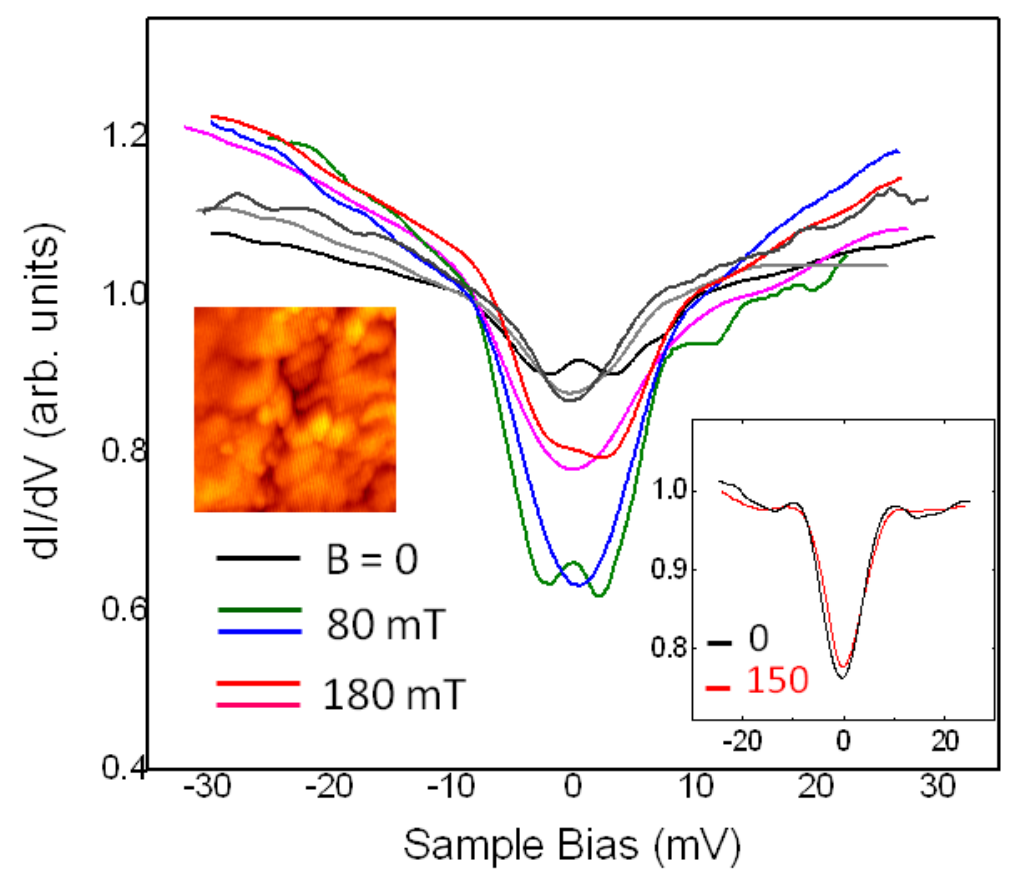

Figure 2. Normalized (to the gap edge) tunneling spectra at $4.2 \mathrm{~K}$ in different magnetic fields, as indicated, on a 14-nm-LCMO/(100)YBCO bilayer, typical of those measured over a $50 \times 50 \mathrm{~nm}^{2}$ region shown by the topographic image (left inset). All spectra exhibit proximity gaps and, in some cases, a signature of a ZBCP. The right inset shows two spectra, one measured after reducing the field to zero (from $180 \mathrm{mT}$ ) and the other after subsequently ramping the field from zero to $150 \mathrm{mT}$.

Another example of the evolution of superconducting-related spectral features in magnetic field is shown in Fig. 3. Here each curve is an average over tens of similar stable spectra all acquired at the same place on LCMO. At zero field we observed a peak slightly shifted toward negative bias and superimposed over an asymmetrical conductance background. In most cases, eliminating the background conductance by subtracting a polynomial fit to the curve excluding the central peak, reduces the peak shift from about 2 $\mathrm{mV}$ to nearly zero bias as shown, for example, for the spectrum acquired at $490 \mathrm{mT}$ (inset 
of Fig. 3a). We therefore refer to such peaks as ZBCPs, although in some spectra a small shift of up to $1 \mathrm{mV}$ remains even after background subtraction, possibly due to asymmetric splitting of the ZBCP (see Supplemental Material) which is too small to be resolved.
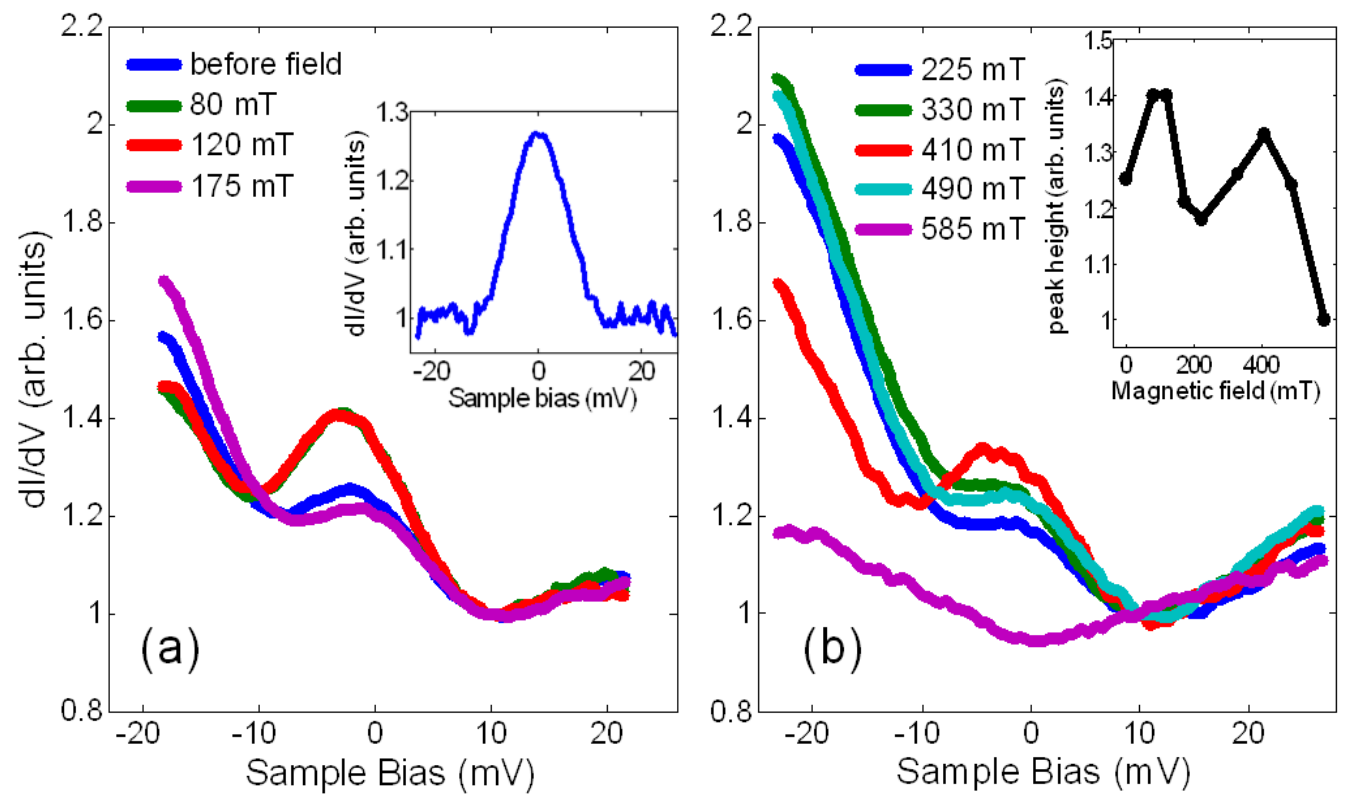

Figure 3: Normalized (to the right dip) tunneling dI/dV versus $V$ spectra at $4.2 \mathrm{~K}$ acquired on a 17-nm-LCMO/(100)YBCO sample showing the evolution of the ZBCP with magnetic field in the regimes 0-175 $\mathrm{mT}(\mathrm{a})$ and 225-585 $\mathrm{mT}(\mathrm{b})$. By subtracting the asymmetric background conductance as explained in the main text, the bias shift of the $Z B C P$ decreases practically to zero, as seen in the inset of (a) for a spectrum acquired at $490 \mathrm{mT}$. The magnitude of the ZBCPs shows a complex dependence on magnetic field, as portrayed by the inset of $(b)$ where the peak height is plotted as a function of magnetic field; the black line is a guide to the eye.

As the field was increased to $80 \mathrm{mT}$ the $\mathrm{ZBCP}$ became more pronounced and the curve hardly varied as the field was increased further to $120 \mathrm{mT}$. However, after further increasing the field to $175 \mathrm{mT}$ the ZBCP diminished in height and became less pronounced than at zero field. This behavior is reminiscent of that of the gap shown in Fig. 1. When 
the field was lowered back to $120 \mathrm{mT}$, the ZBCP increased again (not shown). A nonmonotonic behavior was observed also in the course of the measurement at higher fields, as can be seen in Fig. 3(b). Namely, the small ZBCP at $225 \mathrm{mT}$ increased gradually up to $410 \mathrm{mT}$ but eventually faded away entirely at $\sim 585 \mathrm{mT}$. After lowering the field to zero again, the spectra remained unchanged from those acquired at $585 \mathrm{mT}$. The ZBCP height extracted from each curve as a function magnetic field is shown in the inset of Fig. 3(b).

An effect of the applied field on superconducting-related features was also observed for a 15-nm-LCMO/PCCO bilayer as shown in Fig. 4. Here, we present spectra acquired after zero field cooling (blue and green curves) and after field cycling to $180 \mathrm{mT}$ and back to zero field (reddish curves). All spectra were measured over the same region using identical STM settings. Figure 4(a) shows that the gap feature is about twice as deep after applying the field as compared to the zero field gap, and Fig. 4(b) shows that also the ZBCP has almost doubled after application of the field. We attribute the difference between the spectra acquired before any field was applied and after the field was set back to zero (Fig. 4) to magnetization hysteretic effects that are discussed in the Supplemental Material. ${ }^{15}$ There, we show evidence for dynamic (time-dependent) superconductor PE features in the spectra acquired on LCMO in response to a magnetic field (Fig. S1). The temporal evolution of these features, some of which were not abundantly found, resembles that of magnetization related phenomena observed in thin LCMO films, both in our STM measurements and in a previous ${ }^{24}$ magneto-transport study. This behavior provides further evidence to support our claim that magnetic inhomogeneity plays a key role in generating triplet pairs in LCMO. 

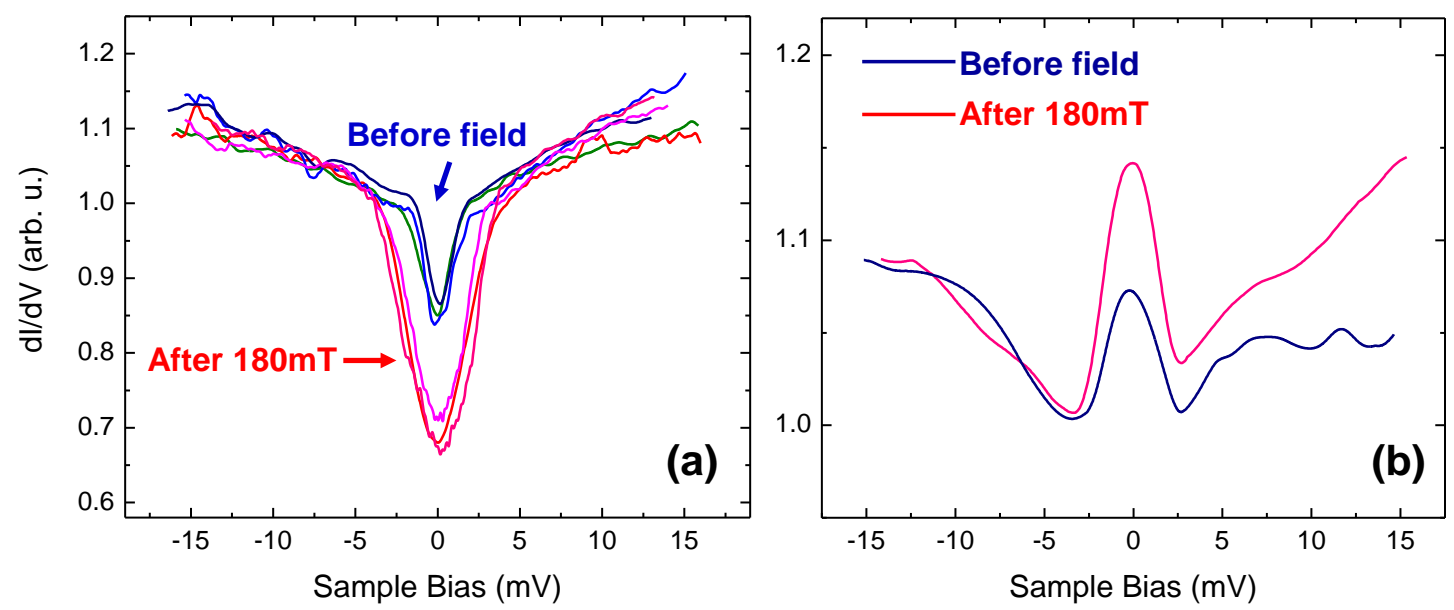

Figure 4: Normalized tunneling dI/dV versus $V$ spectra at $4.2 \mathrm{~K}$ acquired from a $15-\mathrm{nm}$ LCMO/(001)PCCO bilayer at zero filed. The blue and green curves were measured right after zero-field cooling, while the reddish curves right after ramping the field tol $80 \mathrm{mT}$ and back to zero. It is evident that the superconductor-related features, either the gap (a) or $Z B C P(b)$, are enhanced due to the field cycling.

The mechanisms suggested for the induction of a triplet superconductor OP inside a ferromagnet require some form of magnetic inhomogeneity. The corresponding noncollinearity in the magnetization of nearby regions in $\mathrm{F}$ has been predicted theoretically to play an important role in the creation of a triplet pairing component in F-S structures. ${ }^{25,26}$ The magnetic inhomogeneity may be associated, e.g., with domain walls, ${ }^{16}$ or with roughness or strain at the interface that may cause misalignment of the interface magnetization with that of the $\mathrm{F}$ layer interior. ${ }^{17,18}$ Another form of magnetic inhomogeneity specific to (001)YBCO/LCMO interfaces was reported ${ }^{27}$ by Chakhalian et al., where a layer with suppressed magnetization on the LCMO side extends $1 \mathrm{~nm}$ from the interface and is antiferromagnetically coupled to a $2 \mathrm{~nm}$ thick spin polarized layer in the YBCO. Since the magnetizations of both layers are antiparallel, a small magnetic field will cause them to misalign. Such characterization of (100)YBCO/LCMO or $\mathrm{PCCO} / \mathrm{LCMO}$ bilayers, like the one we studied is still lacking.

As we have shown in previous works, ${ }^{11}, 12$ and confirm in the present study, superconductor PE-related features were found in the tunneling spectra (in most cases) on very large areas compared to domain walls of LCMO. We thus assume that some form of wide-spread magnetic inhomogeneity involving misalignment of magnetization in adjacent 
regions is at play in our two systems. Applying a magnetic field to samples with such misalignment will eventually tend to align the magnetizations in both regions along the direction of the applied field. This, however, does not imply that magnetizations in such adjacent regions will tend to align in the same direction at low fields. Specific to our samples, it is important to note that the magnetization easy axis of LCMO lies in-plane, ${ }^{23}$ while the magnetic field was applied perpendicular to the plane. Also, the anisotropy constants and magnetic viscosity in different regions are highly likely to differ. Thus, at finite time, a low field will tend to rotate the magnetization axis out of the plane in adjacent regions but to different extents, depending on the magnetic properties of each region. Since it is likely that the initial magnetizations are predominantly in-plane, a low perpendicular field will increase, in many configurations, the misalignment between the adjacent regions. However, at high enough fields the magnetizations in both regions is expected to align along the applied field, thus reducing the net inhomogeneity. According to this scenario, the application of a magnetic field is expected to result in an initial enhancement of the triplet-pairing state in $\mathrm{F}$ at low fields, followed by its suppression at higher fields. We note that a maximum in the triplet component penetration is anticipated when the magnetizations in the two regions become perpendicular to each other. Since we do not know the exact magnetization configuration throughout the measurement we cannot make a quantitative analysis of the PE as a function of misalignment or size of the magnetic regions. However, as shown by Figs. 1-3, it is clear that the proximity induced gap-like features and ZBCPs, are affected by the magnetic field in a manner consistent with theory, and the field range in which the changes take place are in accord with the magnetization curves presented in the Supplemental Material. ${ }^{15}$ Fig. 3 shows a more complex behavior where at even higher fields an additional enhancement and subsequent reduction of the ZBCP are observed. This behavior can possibly be explained by considering more complex initial magnetization structures. These include, for instance, two regions with magnetization directions making an obtuse angle with the magnetic field or three regions with different magnetic susceptibilities. In any case, at high field (585 mT), where the magnetization is expected to become homogeneous, the ZBCP also disappears and the spectra do not manifest any superconducting related features. Figure 4 focuses on the 
enhancement at low field, which persists for some time due to magnetization viscosity, even after the field is reduced back to zero.

Other scenarios can account for the non-monotonic dependence of the superconductorrelated features on magnetic field. For instance, domain wall motion due to the applied field may locally change the magnetic structure, and thus the magnitude of the tripletpairing PE, in the vicinity of the STM tip. However, the observation of superconducting OP penetration on a much larger scale, ${ }^{11,}{ }^{12}$ possibly due to interface $v s$ F-interior magnetization misalignment, may mask this effect, which is more local in nature. Adjacent regions, either magnetic microdomains or crystallites, possibly separated by anti-phase boundaries, may constitute another form of magnetic inhomogeneity. ${ }^{24,} 28$ These regions are coupled to one another via dipole-dipole interaction and are thus polarized antiparallel to each other at zero field. In response to the application of a magnetic field, the magnetization orientations in these regions will evolve from being in-plane and antiparallel to become aligned parallel to the applied field. Thus, the magnetization non-collinearity will initially increase and subsequently decrease with a corresponding enhancement and suppression of the triplet state.

The changes in ZBCPs due to magnetic field application in our LCMO/YBCO bilayers may be partially associated with corresponding effects observed on bare (110)YBCO ${ }^{29}$ and $\mathrm{Y}_{0.95} \mathrm{Ca}_{0.5} \mathrm{Ba}_{2} \mathrm{Cu}_{3} \mathrm{O}_{7-\delta}{ }^{30}$ Here, changes in $\mathrm{ZBCP}$ height and splitting thereof in response to a magnetic field were attributed to a complex order parameter. The effects observed in these experiments occurred at fields of $\sim 1 \mathrm{~T}$ whereas we observed considerable effects already at $70 \mathrm{mT}$. However, if one takes into account local fields inside the LCMO or close to it, such intensities are attainable. Consequently, if a complex OP emerges in either the YBCO or LCMO, it may also contribute to changes we observe in response to magnetic field, including splitting of ZBCPs (see Supplemental Material).

In summary, we have studied the magnetic field dependence of induced triplet-pairing order parameter in ferromagnetic LCMO films, much thicker than $\xi_{F}$, coupled to either YBCO or PCCO using scanning tunneling spectroscopy. The magnitude of the superconducting-related spectral features, proximity gaps and ZBCPs, showed a nonmonotonic dependence on applied magnetic field, following the anticipated concomitant 
evolution of magnetic inhomogeneity in the LCMO. Our data thus indicate that the local magnetization configuration largely controls the formation of an induced triplet order parameter in the LCMO, and in particular, that magnetic inhomogeneity promotes tripletpairing at S-F interfaces. Further support for this conclusion is provided by the observation of a temporal evolution of proximity-induced superconducting spectral features that resembles that of magnetic-structure related transport effects in LCMO films. Our results have direct implications to the emerging field of superconducting spintronics, ${ }^{20}$ by showing that control over the magnetization configuration is a crucial factor in the design of corresponding devices.

\section{Acknowledgements}

The authors would like to thank Prof. Israel Felner for measurements of the magnetization vs. magnetic field and fruitful discussions. This research was supported in parts by the joint German-Israeli DIP Project (G.K. and O.M.), the United States-Israel Binational Science Foundation (O.M.), the Harry de Jur Chair in Applied Science (O.M.), the Karl Stoll Chair in advanced materials at the Technion (G.K.), the Leverhulme Trust through an International Network Grant (J.W.A.R., M.G.B. and O.M.) and the Royal Society (J.W.A.R.). 


\section{References}

1 L. N. Bulaevskii, A. I. Buzdin, and S. V. Panjukov, Solid State Commun 44, 539 (1982).

A. I. Buzdin, Reviews of Modern Physics 77, 935 (2005).

R. S. Keizer, S. T. B. Goennenwein, T. M. Klapwijk, G. X. Miao, G. Xiao, and A. Gupta, Nature 439, 825 (2006).

M. S. Anwar, F. Czeschka, M. Hesselberth, M. Porcu, and J. Aarts, Phys Rev B 82, 100501 (2010).

M. S. Anwar and J. Aarts, Supercond. Sci. Technol. 24 (2011).

D. Sprungmann, K. Westerholt, H. Zabel, M. Weides, and H. Kohlstedt, Phys Rev B 82, 060505 (2010).

T. S. Khaire, M. A. Khasawneh, W. P. Pratt, Jr., and N. O. Birge, Phys Rev Lett 104, 137002 (2010).

J. W. A. Robinson, J. D. S. Witt, and M. G. Blamire, Science 329, 59 (2010).

C. Visani, Z. Sefrioui, J. Tornos, C. Leon, J. Briatico, M. Bibes, A. Barthelemy, J. Santamaria, and J. E. Villegas, Nat Phys 8, 539 (2012).

K. Dybko, K. Werner-Malento, P. Aleshkevych, M. Wojcik, M. Sawicki, and P. Przyslupski, Phys Rev B 80, 144504 (2009).

Y. Kalcheim, T. Kirzhner, G. Koren, and O. Millo, Phys Rev B 83, 064510 (2011). Y. Kalcheim, O. Millo, M. Egilmez, J. W. A. Robinson, and M. G. Blamire, Phys Rev B 85, 104504 (2012).

Z. Sefrioui, D. Arias, V. Peña, J. E. Villegas, M. Varela, P. Prieto, C. León, J. L. Martinez, and J. Santamaria, Phys Rev B 67, 214511 (2003).

I. Fridman, L. Gunawan, G. A. Botton, and J. Y. T. Wei, Phys Rev B 84, 104522 (2011).

See Supplemental Material at [URL will be inserted by publisher] for sample preparation information, STM measurement process and temporal dependence of spectral features.

A. F. Volkov and K. B. Efetov, Phys Rev Lett 102, 077002 (2009).

M. Eschrig and T. Löfwander, Nat Phys 4, 138 (2008).

J. Linder, M. Cuoco, and A. Sudbø, Phys Rev B 81, 174526 (2010).

F. S. Bergeret, A. F. Volkov, and K. B. Efetov, Phys Rev Lett 86, 4096 (2001).

M. Eschrig, Phys Today 64, 43 (2011).

E. C. Gingrich, P. Quarterman, Y. Wang, R. Loloee, W. P. Pratt, Jr., and N. O. Birge, Phys Rev B 86, 224506 (2012).

M. Ziese, S. P. Sena, and H. J. Blythe, J Magn Magn Mater 202, 292 (1999).

N. M. Nemes, et al., Ieee T Magn 44, 2926 (2008).

M. G. Blamire, B. S. Teo, J. H. Durrell, N. D. Mathur, Z. H. Barber, J. L. M. Driscoll, L. F. Cohen, and J. E. Evetts, J Magn Magn Mater 191, 359 (1999).

M. Houzet and A. I. Buzdin, Phys Rev B 76, 060504 (2007).

A. F. Volkov, F. S. Bergeret, and K. B. Efetov, Phys Rev Lett 90, 117006 (2003).

J. Chakhalian, et al., Nat Phys 2, 244 (2006).

J. Aarts, S. Freisem, R. Hendrikx, and H. W. Zandbergen, Appl Phys Lett 72, 2975 (1998).

R. Beck, Y. Dagan, A. Milner, A. Gerber, and G. Deutscher, Phys Rev B 69, 144506 (2004). 
J. H. Ngai, R. Beck, G. Leibovitch, G. Deutscher, and J. Y. T. Wei, Phys Rev B 82, 054505 (2010). 